\title{
On the Use of Electromagnetic Time Reversal to Locate Faults in Series-Compensated Transmission Lines
}

\author{
R. Razzaghi ${ }^{1}$, Student Member, IEEE, G. Lugrin ${ }^{2}$, M. Paolone ${ }^{1}$, Senior Member, IEEE, and F. Rachidi ${ }^{2}$, Fellow, IEEE \\ ${ }^{1}$ Distributed Electrical System Laboratory (DESL) \\ ${ }^{2}$ Electromagnetic Compatibility Laboratory (EMC) \\ École Polytechnique Fédérale de Lausanne EPFL \\ Lausanne, Switzerland \\ \{reza.razzaghi; gaspard.lugrin; mario.paolone; farhad.rachidi\}@epfl.ch
}

\begin{abstract}
The paper focuses on the extension to seriescompensated multiconductor transmission lines of a new fault location method based on the Electromagnetic Time Reversal (EMTR) theory. The applicability of the EMTR theory to locate faults is first summarized. Then, the paper describes the proposed algorithm to locate faults in multiconductor transmission lines using a single observation point at one of the line terminals. The application of the proposed method to seriescompensated transmission lines is finally illustrated by numerical simulations obtained using the EMTP-RV simulation environment where electromagnetic fault-transients are reproduced with reference to a realistic series-compensated overhead transmission line. The resulting fault location accuracy is quantified and analyzed with reference to different fault types.
\end{abstract}

Index Terms-- Fault location, electromagnetic time-reversal, power systems protection, electromagnetic fault transients, series-compensated transmission lines.

\section{INTRODUCTION}

Accurate fault location is an essential functionality for power systems operators having a large influence on the power system restoration, stability and security in transmission networks and quality of supply in distribution networks.

As summarized in [1], fault location procedures can be classified in two general categories: (i) phasor-based methods (e.g. [2]-[4]) and, (ii) travelling wave-based methods, (e.g. [5]-[13]).

Existing methods belonging to both of the previouslymentioned categories exhibit some drawbacks. In particular, the accuracy of the methods belonging to category (i) suffers from dependency on the system configuration, fault impedance and presence of distributed generations. On the other hand, methods belonging to the second category require measuring systems with large bandwidths and, also, they might predict multiple fault locations (especially when the number of observation points is limited).

Concerning the application field, fault location methods range from distribution networks to transmission systems. Concerning these latters, we can distinguish between fault location methods applied to standard transmission lines and those that have been specifically developed for seriescompensated lines ${ }^{1}$. However, the use of series capacitors for line reactance compensation introduces major difficulties in the fault location processes adopted for these specific lines (e.g. [14]-[16]).

Fault location in series-compensated transmission lines has a more crucial role since these type of lines are designed to link distant nodes among which high amount of power is usually transferred. As summarized in [16], fault location methods for series-compensated transmission lines are based on one or multiple-end measurements and, in general, are based on post-fault impedance assessment.

Within this context, this paper aims at extending the application of the so-called time-reversal process to the problem of the fault location in series-compensated transmission lines. The time reversal process was developed firstly in the field of acoustics [17]-[20] and was more recently applied to electromagnetics (e.g. [21]-[24]). In what follows, we will make reference to the time reversal process applied to electromagnetic transients using the acronym EMTR (Electromagnetic Time Reversal).

In a previous study, the authors of this paper have demonstrated the applicability of the EMTR technique to locate faults in a single-conductor transmission line using

\footnotetext{
${ }^{1}$ As known series-compensated transmission lines provide many benefits such as: increase of transmission capability, improvement of power systems stability, direct power flow control, with substantial environmental and economic benefits. These transmission systems are expected to play a significant role in modern electrical transmission infrastructures.
} 
either multiple observation points [25], or a single observation point [26]. The present paper aims at extending the applicability of such a fault location technique to the case of series-compensated multiconductor transmission lines using a single observation point.

The structure of the paper is as follows. Section II describes the basic concept of the EMTR theory. In Section III, the EMTR-based fault location algorithm is presented for the case of multiconductor transmission lines where post-fault electromagnetic transients are measured in a single observation point. Section IV illustrates the application of the proposed method using EMTP-simulated cases referring to a multiconductor transmission line. Section V concludes the paper with a summary and conclusions.

\section{BASIC CONCEPT OF THE EMTR THEORY}

In this Section, we examine the properties of the transmission line wave equations under time reversal $[25,26]$. The time reversal operator corresponds to the change of the sign of the time, i.e. to the following transformation

$$
t \mapsto-t
$$

An equation is defined as 'time-reversal invariant' if it is invariant under the application of the time-reversal operator. The voltage wave equation for a multiconductor, lossless transmission line reads

$$
\frac{\partial^{2}}{\partial x^{2}} \mathbf{U}(x, t)-\mathbf{L}^{\prime} \cdot \frac{\partial^{2}}{\partial t^{2}} \mathbf{U}(x, t)=0
$$

where $\mathbf{U}(x, t)$ is a vector containing the phase voltages at position $x$ and time $t, \mathbf{L}^{\prime}$ and $\mathbf{C}^{\prime}$ are the per-unit-length parameter matrices of inductance and capacitance of the line, respectively. Time reversing (2) yields:

$$
\frac{\partial^{2}}{\partial x^{2}} \mathbf{U}(x,-t)-\mathbf{L}^{\prime} \mathbf{C}^{\prime} \frac{\partial^{2}}{\partial t^{2}} \mathbf{U}(x,-t)=0
$$

Therefore, if $\mathbf{U}(x, t)$ is a solution of the wave equation, then $\mathbf{U}(x,-t)$ is a solution too. In other words, as described in [18][20] for ultrasonic waves, the wave equation is invariant under a time-reversal transformation if there is no absorption during propagation in the medium. In our specific application, this hypothesis is rigorously satisfied only if the transmission line is lossless. However, since power network transmission lines are generally characterized by small values for the longitudinal resistance, the applicability of EMTR to this case could also be considered [26].

In practical implementations, a signal $s(x, t)$ is necessarily measured only during a finite period of time from an initial time selected here as the origin $t=0$ to a final time $t=T$, where $T$ is a pre-defined observation window long enough to damp out all the transients in $s(x, t)$. To make the argument of the time-reversed variables positive for the duration of the signal, we will add, in addition to time reversal, a time delay equal to $T$, namely:

$$
s(x, t) \mapsto s(x, T-t)
$$

Another peculiarity of the EMTR is that it is particularly efficient when the system is limited in space [20], i.e. when the domain is characterized by boundary conditions other than perfectly absorbing ones. This feature fits the conditions associated with travelling waves in electrical power systems composed by transmission lines terminated by generic power components (transformers, power converters, etc.).

\section{EMTR-BASED FAULT LOCATION ALGORITHM}

The flow-chart shown in Fig. 1 illustrates the step-by-step fault location procedure based on EMTR. As it can be seen, the proposed procedure requires the knowledge of the network topology as well as its parameters. Such knowledge is used to build a corresponding network model. Fault transients, $s_{i}(t)$ (with $i=1,2,3$ for a three-phase system) are assumed to be recorded at a given observation point located inside the part of the network with the same voltage level comprised between transformers.

The transient signal initiated by the fault is assumed to be recorded within a specific time window, namely:

$$
s_{i}(t), \quad t \in\left[t_{f}, t_{f}+T\right]
$$

where $t_{f}$ is the fault triggering time, and $T$ is the recording time window above defined.

The unknowns of the problem are: (i) the fault type, (ii) location and (iii) its impedance. Concerning the fault type, we assume that the fault location procedure will operate after the relay maneuver. Therefore, the nature of the fault (single or multi-phase) is assumed to be known by the 'trip signal' sent from the protective relay towards the fault locator. Concerning the fault location, we assume a set of a priori locations $x_{f, m}, m=1, \ldots, K$ for which the EMTR procedure is applied. It is worth observing that, in order to reduce the number of guessed fault locations, a preliminary guessed fault location calculated by modern protective relays could be utilized. Then, the proposed method could be applied to further improve the accuracy of the fault location obtained by other systems.

Concerning the fault impedance, for all the guessed fault locations, an a priori value of the fault resistance, $R_{x_{f}}$, is assumed. As it will be shown in Section IV, different guessed values of $R_{x_{f}}$ do not affect the fault location accuracy. Then, the recorded signal is reversed in time (equation (1)) and backinjected from one the observation point into the system for each $x_{f, m}$. 


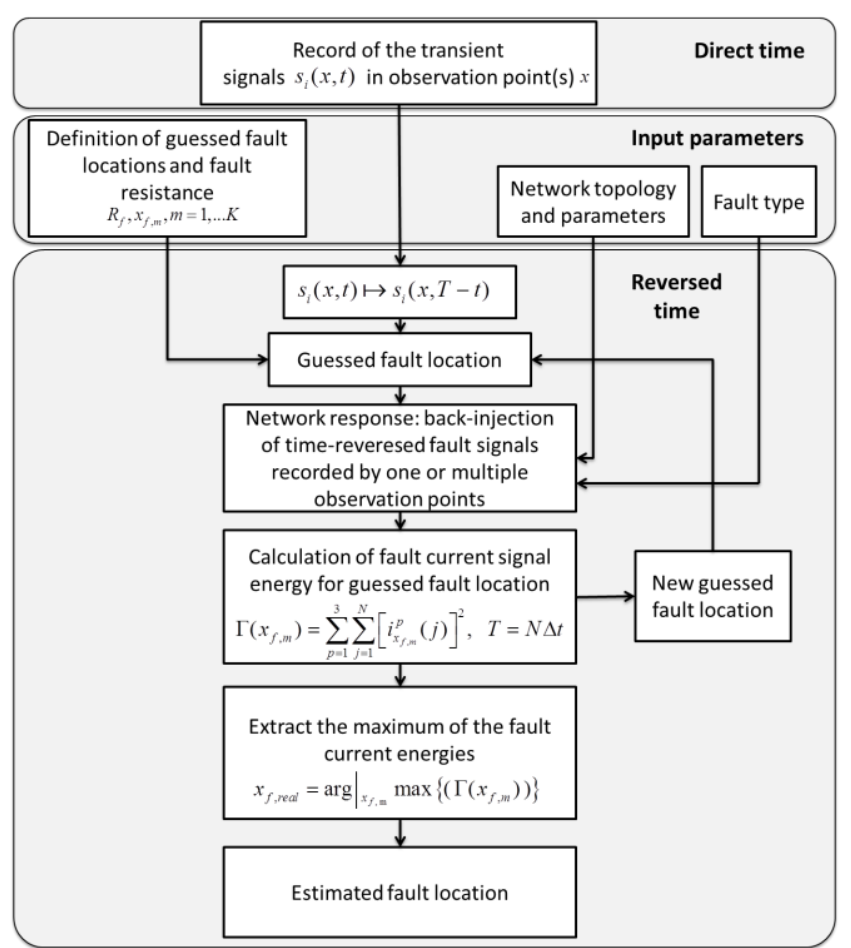

Fig. 1. Flow-chart of the proposed fault location method. Adapted from [26].

As mentioned previously, in order to make the argument of the time-reversed variables positive for the duration of the signal, we add, in addition to the time reversal, a time delay equal to the duration of the recording time $T$ :

$$
\begin{aligned}
& \hat{t}=\left(T+t_{f}\right)-t \\
& \bar{s}(\hat{t}), \hat{t} \in[0, T]
\end{aligned}
$$

For each of the guessed fault location, we can compute the energy of the signal that corresponds to the currents flowing through the guessed fault location as:

$$
\Gamma\left(x_{f, m}\right)=\sum_{p=1}^{3} \sum_{j=1}^{N}\left[i_{x_{f, m}^{p}}^{p}(j)\right]^{2}, T=N \Delta t
$$

where $N$ is the number of samples, $\Delta t$ the sampling time, and $p$ indicates the different phases $(a, b, c)$ of the line. According to the EMTR method, the energy given by (8) is maximized at the position of the fault [26]. Thus, the maximum of the calculated signal energies will indicate the real fault location:

$$
x_{f, \text { real }}=\left.\arg \right|_{x_{f, \mathrm{~m}}} \max \left\{\left(\Gamma\left(x_{f, m}\right)\right)\right\}
$$

\section{APPLiCATION EXAMPLE}

To examine the performance of the proposed method, an application example is considered by making reference to a three-conductor series-compensated transmission line. The line length is $200 \mathrm{~km}$ and it has been modeled by means of a constant-parameter (CP) model implemented within the EMTP-RV simulation environment [27]-[29]. The seriescompensation is done in the center of the transmission line to achieve a compensation degree of 50\%. The relevant line parameters are the following:

- Positive sequence impedance: $0.03293+\mathrm{j} 0.3184 \Omega / \mathrm{km}$

- Positive sequence capacitance: $0.01136 \mu \mathrm{F} / \mathrm{km}$

- Zero sequence impedance: $0.2587+\mathrm{j} 1.1740 \Omega / \mathrm{km}$

- Zero sequence capacitance: $0.00768 \mu \mathrm{F} / \mathrm{km}$.

The line is assumed to be terminated at both ends on power transformers which, for signals characterized by highfrequency spectrum content, can be replaced by high impedances ( $100 \mathrm{k} \Omega$ in this study).

The supply of the line is provided by a three-phase AC voltage source placed at $x=0$. A schematic representation of the system is shown in Fig 2. In this figure, OP1, OP2, and OP3 are observation points corresponding to each conductor of the transmission line where voltage transients are recorded.

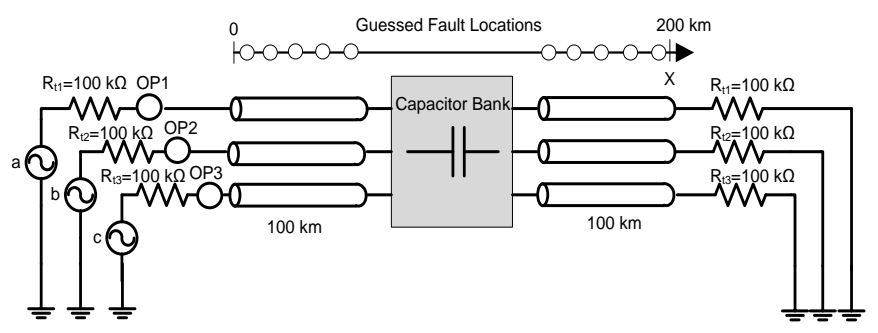

Fig. 2. Schematic representation of the series-compensated three-condcutor transmission line system implemented in the EMTP-RV.

To examine the performance of the proposed method, three fault cases are considered: (i) a three-phase-to-ground fault at $x_{f}=75 \mathrm{~km}$, (ii) a double phase-to-ground fault at $x_{f}=35 \mathrm{~km}$ and (iii) a single-phase-to-ground fault at $x_{f}=25 \mathrm{~km}$. All these three fault cases are assumed to be solid faults.

A fault occurrence generates transient signals in all three conductors of the transmission line. These transient signals are recorded in a single observation point located at $x=0$ in all the three conductors of the line (OP1, OP2, and OP3). After time reversing the recorded signals, in agreement with the proposed procedure, and by assuming the knowledge of the fault type, the same fault type is realized in time-reversed state and the position of the guessed fault location is moved along the line assuming, for the fault impedance, an a priori fixed value. It is worth observing that, as it will be shown next, the value of the a priori guessed fault resistance does not affect the accuracy of the proposed fault location method.

Figures 3, 4, and 5 show the energy of the current flowing through the guessed fault points for the three-phase-to-ground fault, double-phase-to-ground fault, and single-phase-toground fault, respectively. For each case, the energy values 
are normalized to the corresponding peak value. These figures illustrate the calculated normalized fault current energies for two a priori guessed values of the fault resistance, namely $1 \Omega$ and $10 \Omega$. In order to evaluate the accuracy of the proposed method, the position of the guessed fault location was varied every $1 \mathrm{~km}$ near to the real fault location.

As it can be seen, the proposed method is remarkably effective in identifying the fault location for all the three fault cases (the maximum peak of the fault current energy is obtained at the real fault location). Additionally, it appears robust against the a priori assumed fault impedance.

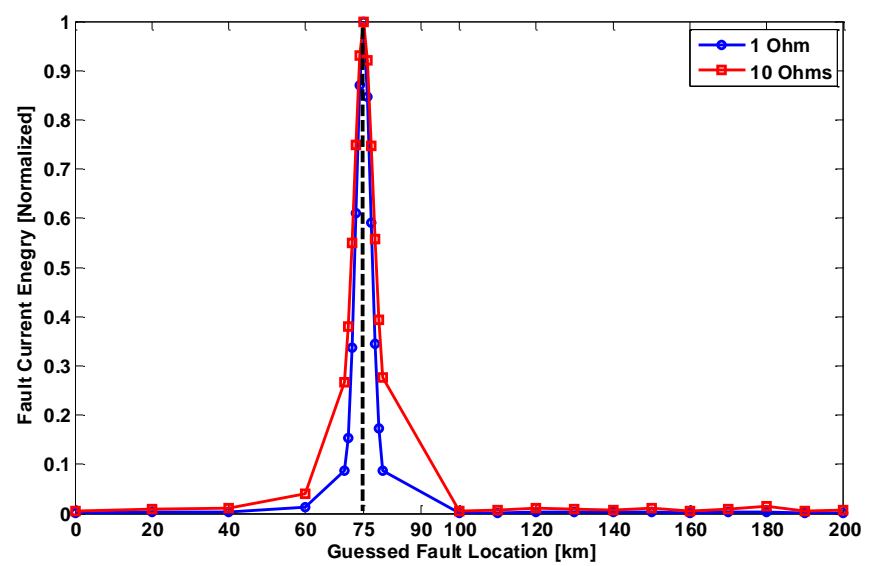

Fig.3. Normalized energy of the fault current as a function of the guessed fault location and for different guessed fault resistance values (i.e., 1 and 10 Ohms). The real fault is a three-phase-to-ground fault at $x_{\mathrm{f}}=75 \mathrm{~km}$.

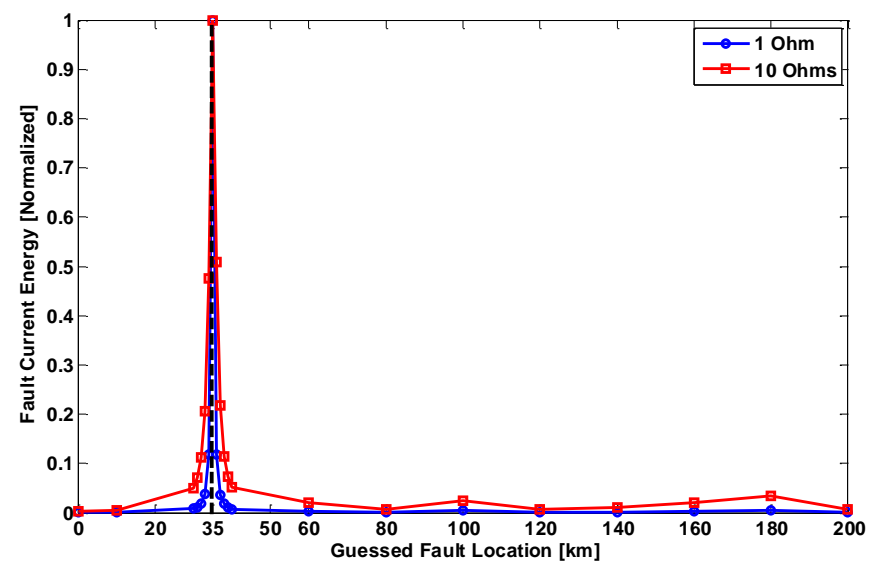

Fig. 4. Normalized energy of the fault current as a function of the guessed fault location and for different guessed fault resistance values (i.e., 1 and 10 Ohms). The real fault is a double-phase-to-ground fault at $x_{\mathrm{f}}=35 \mathrm{~km}$.

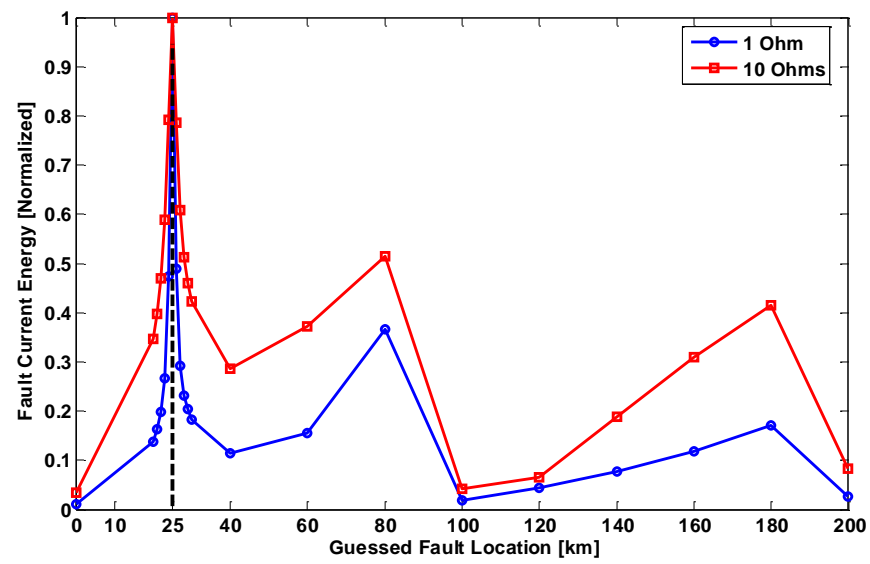

Fig.5. Normalized energy of the fault current as a function of the guessed fault location and for different guessed fault resistance values (i.e., 1 and 10

Ohms). The real fault is a single-phase-to-ground fault at $x_{\mathrm{f}}=25 \mathrm{~km}$.

\section{CONCLUSION}

In this paper, a new fault location method based on the electromagnetic time-reversal theory, originally proposed by the authors in $[25,26]$, was extended to the case of multiconductor series-compensated transmission lines. The application of the EMTR to locate faults in a power network is carried out in three steps: (1) measurement of the faultoriginated electromagnetic transient in a single observation point, (2) simulation of the back-injection of the timereversed measured fault signal for different guessed fault locations using a network model capable of representing traveling-waves, and (3) determination of the fault location by finding, in the network model, the point characterized by the highest energy concentration associated with the backinjected time-reversed fault current. One of the main advantages of the developed EMTR-based fault location method is that it requires, in general, only one observation point. Additionally, the proposed method also exhibits a robust behavior against the a priori assumed fault impedance.

The resulting fault location accuracy and robustness appear to be very promising for the case of seriescompensated transmission lines.

In view of the relatively modest computational requirements, the proposed method could be implemented in intelligent Electronic Devices (IED) as a real-time fault locator system or could also been utilized in offline mode.

\section{ACKNOWLEDGEMENTS}

The authors thankfully acknowledge the constructive comments received from the anonymous reviewers of the paper, which motivated us to extend the applicability of the proposed fault location method to series-compensated transmission lines. 


\section{REFERENCES}

[1] IEEE Std C37.114, "IEEE guide for determining fault location on AC transmission and distribution lines," 2004.

[2] M. S. Sachdev and R. Agarwal, "A technique for estimating transmission line fault locations from digital impedance relay measurements," IEEE Trans. Power Del., vol. 3, no. 1, pp. 121-129, Jan. 1988.

[3] K. Srinivasan and A. St.-Jacques, "A new fault location algorithm for radial transmission lines with loads," IEEE Trans. Power Del., vol. 4, no. 3, pp. 1676-1682, Jul. 1989.

[4] A. A. Girgis, D. G. Hart, and W. L. Peterson, "A new fault location technique for two- and three-terminal lines," IEEE Trans. Power Del., vol. 7, no. 1, pp. 98-107, Jan. 1992.

[5] G. B. Ancell and N. C. Pahalawatha, "Maximum likelihood estimation of fault location on transmission lines using travelling waves," IEEE Trans. Power Del., vol. 9, no. 2, pp. 680-689, Apr. 1994.

[6] O. Chaari, M. Meunier, and F. Brouaye, "Wavelets: A new tool for resonant grounded power distribution systems relaying," IEEE Trans. Power Del., vol. 11, no. 3, pp. 1301-1308, Jul. 1996.

[7] F. H. Magnago and A. Abur, "Fault location using wavelets," IEEE Trans. Power Del., vol. 13, no. 4, pp. 1475-1480, Oct. 1998.

[8] F. H. Magnago and A. Abur, "A new fault location technique for radial distribution systems based on high frequency signals," in Proc. IEEEPower Eng. Soc. Summer Meeting, vol. 1, pp. 426-431, Jul. 18-22, 1999.

[9] D. W. P. Thomas, R. E. Batty, C. Christopoulos, and A. Wang, "A novel transmission-line voltage measuring method," IEEE Trans. Instrum. Meas., vol. 47, no. 5, pp. 1265-1270, Oct. 1998.

[10] Feng Yan, Zhiye Chen, Zhirui Liang, Yinghui Kong, Peng Li, "Fault location using wavelet packets", Proc. of Int. Conf. on Power System Technology, PowerCon 2002, Vol. 4, pp. 2575 - 2579, 13-17 Oct. 2002.

[11] A. Borghetti, S. Corsi, C. A. Nucci, M. Paolone, L. Peretto, and R. Tinarelli, "On the use of continuous-wavelet transform for fault location in distribution power networks," Elect. Power Energy Syst., vol. 28, pp. 608-617, 2006.

[12] A. Borghetti, M. Bosetti, M. Di Silvestro, C.A. Nucci and M. Paolone, "Continuous-Wavelet Transform for Fault Location in Distribution Power Networks: Definition of Mother Wavelets Inferred from Fault Originated Transients", IEEE Trans. on PWRS, vol. 23, No. 2, pp.380388, may 2008.

[13] A. Borghetti, M. Bosetti, C.A. Nucci, M. Paolone, A. Abur, "Integrated Use of Time-Frequency Wavelet Decompositions for Fault Location in Distribution Networks: Theory and Experimental Validation", IEEE Trans. Power Del., vol. 25, issue 4, Oct. 2010, pp. 3139 - 3146.

[14] C. Yu, C. Liu, S. Yu, and J. Jiang, "A new PMU-based fault location algorithm for series compensated lines," IEEE Trans. Power Del., Vol. 17, no. 1, January 2002.

[15] P. Jena, A. K. Pradhan, "A positive-sequence directional relaying algorithm for series-compensated line," IEEE Trans. Power Del., vol. 25, no. 4, October 2010.

[16] J. Izykowski, E. Rosolowski, P. Balcerek, M. Fulczyk, M. M. Saha, "Fault location on double-circuit series-compensated lines using two- end unsynchronized measurements," IEEE Trans. Power Del., vol. 26, no. 4, October 2011.

[17] M. Fink, C. Prada, F. Wu and D. Cassereau, "Self focusing with time reversal mirror in inhomogeneous media", IEEE Ultrason. Symp., pp. 681-686, 1989.

[18] M. Fink, "Time reversal of ultrasonic fields. I. Basic principles", IEEE Trans. on Ultrasonics, Ferroelectrics and Frequency Control, vol. 39, issue 5, Sept. 1992, pp. $555-566$.

[19] F. Wu, J.-L. Thomas, M. Fink, "Time reversal of ultrasonic fields. II: Experimental results", IEEE Trans. on Ultrasonics, Ferroelectrics and Frequency Control, vol. 39, issue 5, Sept. 1992, pp: $567-578$.

[20] D. Cassereau, M. Fink, "Time-reversal of ultrasonic fields. III. Theory of the closed time-reversal cavity", IEEE Trans. on Ultrasonics, Ferroelectrics and Frequency Control, vol. 39, issue 5, Sept. 1992, pp: $579-592$.

[21] G. Lerosey, J. de Rosny, A. Tourin, A. Derode, G. Montaldo, M. Fink, "Time reversal of electromagnetic waves", Phys. Rev. Lett. 92, pp. 193904-193901-193903, 2004.

[22] D. Liu; G. Kang; L. Li; Y. Chen; S. Vasudevan, W. Joines, Q. Huo Liu, J. Krolik, L. Carin, "Electromagnetic time-reversal imaging of a target in a cluttered environment", IEEE Trans. Antennas Propagat., vol. 53, pp. 3058-3066, 2005.

[23] H. Zhai, S. Sha, V. K. Shenoy, S. Jung, M. Lu, K. Min, S. Lee, D.S. $\mathrm{Ha}$, "An electronic circuit system for time-reversal of ultra-wideband short impulses based on frequency-domain approach", IEEE Transactions on Microwave Theory and Techniques,, vol. 58, issue: 1, pp. $74-86,2010$.

[24] N. Mora, F. Rachidi, M. Rubinstein, "Application of the time reversal of electromagnetic fields to locate lightning discharges", Journal of Atmospheric Research, Vol. 117, pp. 78-85, 2012.

[25] H. M. Manesh, G. Lugrin, R. Razzaghi, C. Romero, M. Paolone, F. Rachidi, "A new method to locate faults in power networks based on electromagnetic time reversal", Proc. of 13th IEEE International Workshop on Signal Processing Advances in Wireless Communications, SPAWC, June 2012.

[26] R. Razzaghi, G. Lugrin, H. Manesh, C. Romero, M. Paolone, F. Rachidi, "An efficient method based on the electromagnetic time reversal to locate faults in power networks," accepted in IEEE Trans. Power Del., 2013. DOI: 10.1109/TPWRD.2013.2251911.

[27] H. W. Dommel: "Digital computer solution of Electromagnetic Transiens in single and multiphase networks", IEEE Transactions, Vol. PAS-88, pages 388-399, April 1969.

[28] J. Mahseredjian, S. Lefebvre and X.-D. Do, "A new method for timedomain modelling of nonlinear circuits in large linear networks", Proc. of 11th Power Systems Computation Conference PSCC, August 1993.

[29] J. Mahseredjian, S. Dennetière, L. Dubé, B. Khodabakhchian and L. Gérin-Lajoie: "On a new approach for the simulation of transients in power systems”. Electric Power Systems Research, Volume 77, Issue 11, September 2007, pp. 1514-1520. 